Portland State University

PDXScholar

Spring 7-12-2018

\title{
Framing Physical Activity: Weight Control Frames and Physical Activity Motivation
}

Kristin Spurkland

Portland State University

Follow this and additional works at: https://pdxscholar.library.pdx.edu/open_access_etds

Part of the Community Health Commons, and the Life Sciences Commons Let us know how access to this document benefits you.

\section{Recommended Citation}

Spurkland, Kristin, "Framing Physical Activity: Weight Control Frames and Physical Activity Motivation" (2018). Dissertations and Theses. Paper 4448.

https://doi.org/10.15760/etd.6332

This Thesis is brought to you for free and open access. It has been accepted for inclusion in Dissertations and Theses by an authorized administrator of PDXScholar. Please contact us if we can make this document more accessible: pdxscholar@pdx.edu. 
Framing Physical Activity: Weight Control Frames and Physical Activity Motivation

by

Kristin Spurkland

A thesis submitted in partial fulfillment of the requirements for the degree of

Master of Science

in

Health Studies

Thesis Committee:

Gary R. Brodowicz, Chair

Randy Miller

Claire Wheeler

Portland State University

2018 


\begin{abstract}
Public health institutions and popular media frequently frame weight loss and weight control as primary benefits of physical activity. This exploratory, descriptive study examined how respondents rated statements exemplifying three physical activity frames: a weight control frame, a medical frame, and an active embodiment frame. An anonymous, online survey was conducted in March 2018; respondents rated frame statements in terms of inspiring motivation to engage in physical activity, and in terms of perceived credibility. They also provided anthropometric data and physical activity data. Data were analyzed for the entire sample as well as stratified by multiple variables, including body mass index, waist circumference, age, and physical activity levels.

Overall, the weight control frame was rated the lowest in terms of motivation, and rated moderately in terms of credibility. The active embodiment frame was highly rated in terms of motivation, but did not rate highly in terms of credibility. The medical frame was rated most credible overall, while achieving moderate motivation ratings. A “credibility/motivation gap" was identified when frames were rated highly on one scale (credibility or motivation), but not the other. These findings have implications for how physical activity is framed in public health messaging, and suggest that, as no single frame dominated both the motivation and credibility ratings, a multi-frame approach may have greater success in motivating people to engage physical activity than does the current, weight-control dominant approach.
\end{abstract}




\section{Table of Contents}

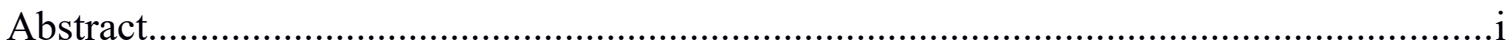

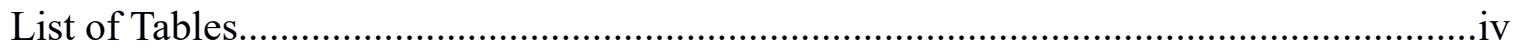

Chapter I

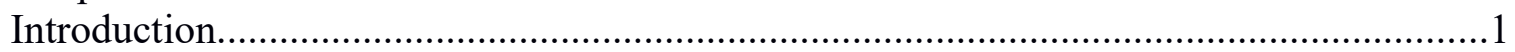

Statement of the Problem and Significance of Study................................................ 1

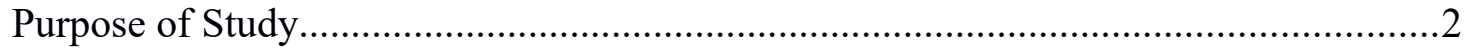

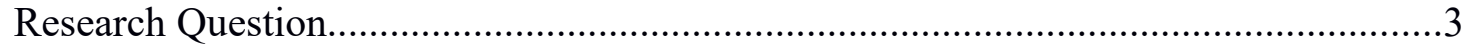

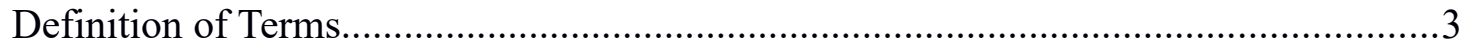

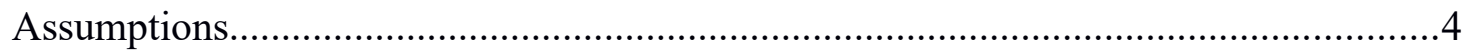

Chapter II

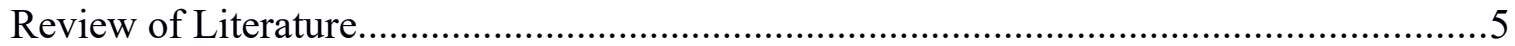

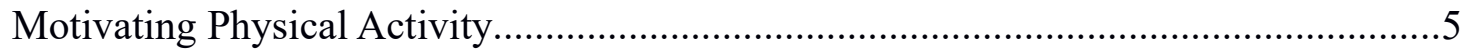

Fatness as a Measure of Health.........................................................................6

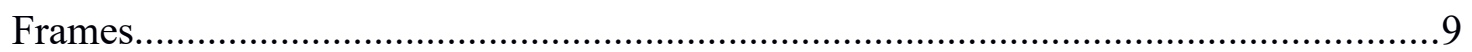

Physical Activity, Weight Control, and False Hope Syndrome...................................10

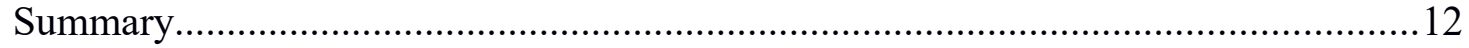

Chapter III

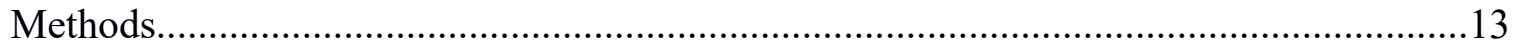

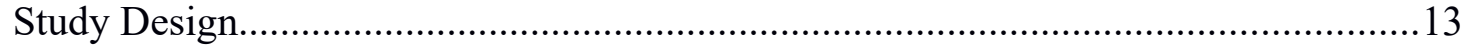

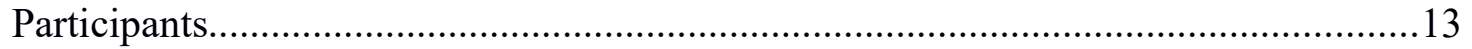

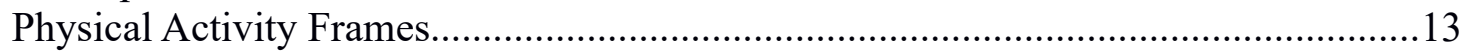

General Procedures........................................................................................... 15

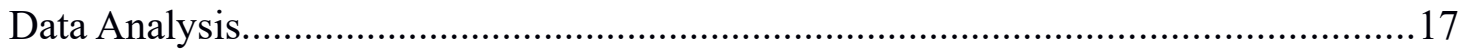

Chapter IV

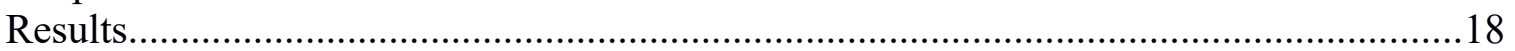

Demographic and Anthropometric Characteristics................................................. 18

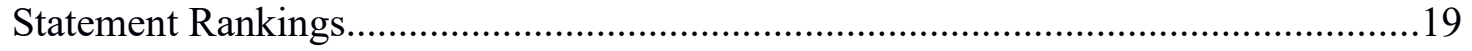

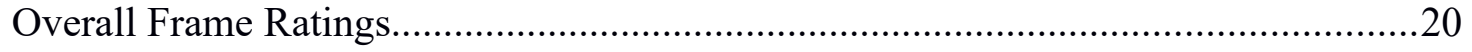

Frame Ratings Stratified by PA Levels, Age, and Gender........................................20

Frame Ratings Stratified by BMI, WCirc, and Weight Status..................................22

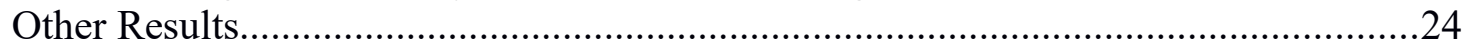

Chapter V

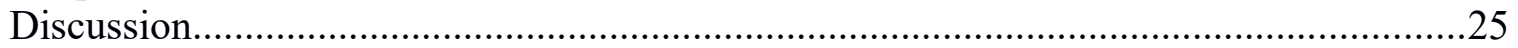

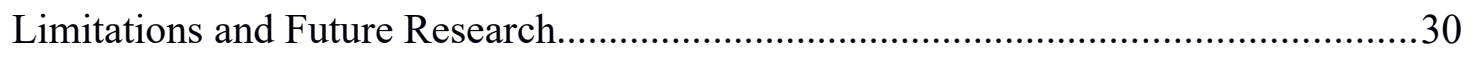

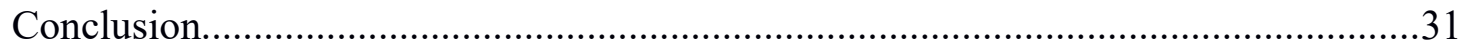




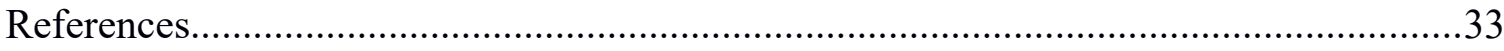

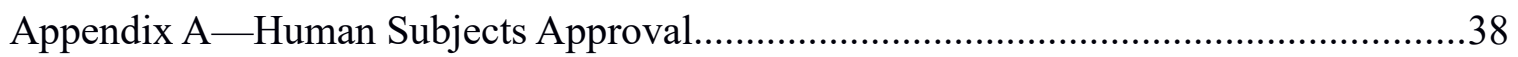

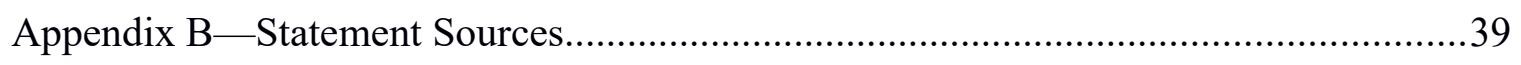

Appendix C_-Framing Physical Activity Survey............................................... 41

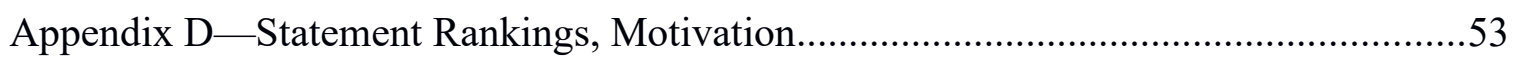

Appendix E—Statement Rankings, Credibility........................................................54

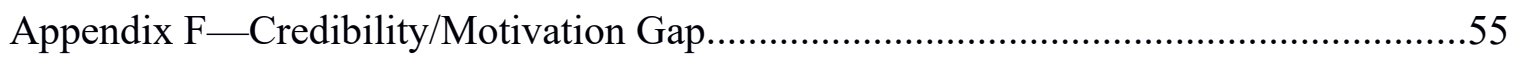




\section{List of Tables}

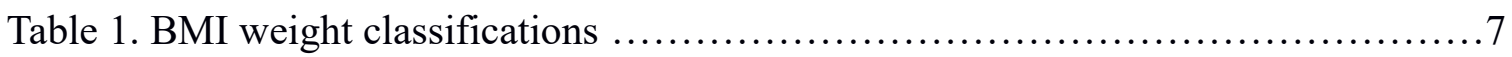

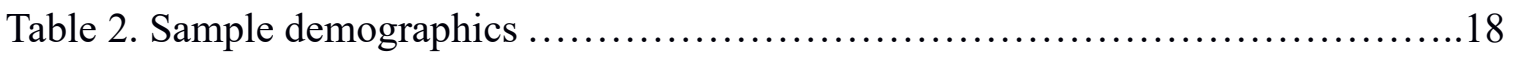

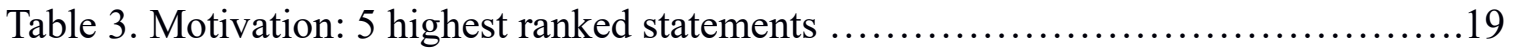

Table 4. Credibility: 5 highest ranked statements ..............................20

Table 5. Frame ratings, stratified by PA, age, and gender ...........................21

Table 6. Frame ratings, stratified by BMI, WCirc, and weight status ...................23 


\section{Chapter I}

\section{Introduction}

\section{Statement of the Problem and Significance of Study}

Despite public health campaigns and popular media messaging delineating the many benefits of physical activity — including improved outcomes related to coronary heart disease, stroke, some cancers, type 2 diabetes, osteoporosis, depression, and the ability to successfully engage in activities of daily living (United States Department of Health Human Services, 2008) — only about one in five adults meet the 2008 federal guidelines for physical activity (Centers for Disease Control and Prevention, 2014). In addition to their association with reduced health outcomes, low physical activity (PA) levels are linked to the global rise in overweight and obesity. Defined by the World Health Organization (WHO) as "abnormal or excessive fat accumulation that may impair health" (World Health Organization, 2018), overweight and obesity are associated with increased risk of hypertension, dyslipidemia, Type 2 diabetes, coronary heart disease, stroke, osteoarthritis, reduced quality of life, increased mental illness, and overall increased mortality (Centers for Disease Control and Prevention, 2015b).

These twin concerns of low PA levels and rising levels of overweight/obesity are intertwined via public health and popular media messaging that promotes weight loss, weight control, and body shaping as the primary benefits of PA (Segar, Eccles, \& Richardson, 2011). The return page for a February 2018 Google search of the phrase "benefits of physical activity" exemplifies this emphasis on weight loss/body shaping. Featured at the top of the search return page was a box in which "Control Your Weight" 
was the first benefit listed. In the same box was a link to the Centers for Disease Control and Prevention's "Physical Activity and Health" page. The page opens with a list of the benefits of physical activity, the first item listed being "control your weight" (Centers for Disease Control and Prevention, 2015a). A Mayo Clinic article titled "Exercise: 7 Benefits of Regular Physical Activity" places "Exercise controls weight" at the top of the list (Mayo Clinic, 2016). The LIVESTRONG.com fitness home page yields an image of an exceptionally lean and muscled woman, and a large side bar, occupying a full third of the page, titled "LIVESTRONG.com Weight Loss Tools", rendering weight loss the dominant message on the fitness home page. Finally, multiple Google autocompletes of the phrase "exercise to...", conducted over several months, inevitably yielded results in which weight loss and body shaping topics were the most frequently returned ("exercise to lose weight", "exercise to lose belly fat", "exercise to lose fat", "exercise to shape $a b s ")$. Thus, conducting an internet search on the benefits of physical activity and exercise results in receiving the pervasive message that the primary benefits are weight and body shape related.

Based on current levels of PA in the United States, it appears that the current approaches to PA promotion have been minimally successful. As increased levels of PA are a national health goal (Office of Disease Prevention and Health Promotion, 2018), a better understanding of the effectiveness of the dominant PA messaging is needed.

\section{Purpose of Study}

The purpose of this study was to examine how respondents rated statements exemplifying three PA frames (a weight control frame, a medical frame, and an active 
embodiment frame) in terms of inspiring motivation to engage in PA, and in terms of perceived credibility; given its dominance in public health and popular media messaging, responses to the weight control frame were of primary interest.

\section{Research Question}

Does the framing of weight control as a primary benefit of PA motivate adults to engage in PA? Secondary questions: Do adults find the weight control frame credible? How does the active embodiment frame compare to the weight control frame in terms of motivation and credibility?

My prediction was that PA advocacy closely linking PA to weight control would have reduced efficacy in motivating PA as compared to advocacy taking a weight neutral stance. I also predicted that statements linking PA to weight control would not be rated as credible, especially among respondents who fell into the overweight/obese BMI categories.

\section{Definition of Terms}

Physical activity and exercise: While the fields of fitness and exercise science make a distinction between "physical activity" (all voluntary muscular activity) and "exercise" (planned muscular activity with specific goals of increased strength and/or fitness)

(Powers \& Howley, 2012), public health and popular media messaging use the terms interchangeably, with "physical activity" being the more frequently utilized term. For the purposes of this paper, I followed the protocols of the institutions and organizations utilized, and did not make a distinction between the two modalities. For ease of reading, I used the acronym "PA" throughout do denote "physical activity and exercise". 
Standard physical activity recommendations for adults: For improved health outcomes, 150 minutes of moderate-intensity aerobic activity every week and strength training for all major muscle groups at least 2 days per week are recommended (United States Department of Health Human Services, 2008).

Fat: Following in the footsteps of sociologist Abigail Saguy, I use "fat" as a neutral descriptor. While still read as pejorative by many, "fat" does not carry the same clinical and medical connotations as the words "overweight" and "obese"; additionally, "fat" as a neutral adjective is in the process of being re-claimed by members of the fat rights movement (Saguy, 2013).

Stigma: A stigma is "an attribute that is deeply discrediting" and an "undesired differentness" from what those without the stigma (Goffman, 1963, p. 3). Stigmas allow observers to define, categorize, and make assumptions about a person upon encountering them for the first time. Over time, stigmatized people may internalize the stigma, believing that they fail to live up to what they should be (Goffman, 1963).

\section{Assumptions}

1. Respondents provided honest, accurate data to the best of their ability.

2. The sample in this study is representative of the larger adult population.

3. My conceptualization of PA frames accurately represented PA messaging as experienced by the greater population. 


\section{Chapter II}

\section{Review of Literature}

\section{Motivating Physical Activity}

Understanding what motivates individuals to engage in PA (when there are so many ways other ways that they could spend their free time) is central to development of successful PA promotions (Sherwood \& Jeffery, 2000). Obtaining and maintaining health is a significant contributor to adults' reported motivation to engage in regular PA (Segar, Eccles, \& Richardson, 2011; Rochholz \& Zidon, 2003; Trujillo, Brougham, \& Walsh, 2004). Other highly ranked motivators include developing strength and endurance, weight management, enjoyment of the specific PA activity, and improved appearance (Segar et al., 2011; Rochholz \& Zidon, 2003). Motivation related to physical appearance and weight loss tends to decrease with age, while motivation related to health and functioning tend to increase (Schuler et al., 2004; Trujillo et al., 2004).

However, motivation towards PA doesn't necessarily translate into participation in PA; research shows that different goals are associated with different levels of PA participation. For example, a study of healthy middle-aged women (age 40-60 years) that examined the association between stated exercise goals and actual exercise participation, Segar et al. (2011) found that those women who identified "quality of life" as their superordinate goal ("big goals" that reflect the individual's idealized self; goals are central to motivation) exercised $34 \%$ more than women who identified "weight loss/appearance" as their superordinate goal, and 25\% more than women whose superordinate to goal was to improve their current health status. Perhaps paradoxically, 
less than $25 \%$ of the study's subjects identified quality of life as their superordinate goal; health-related and weight loss goals were favored by the majority. The study's authors proposed that quality of life's low ranking as a goal may be a reflection of the way exercise and PA are "branded", noting that PA is promoted primarily for its health and weight control benefits. They further suggested that women may not consider PA as a pathway to improved daily quality of life, as they have not been socialized to do so. Socialization in this context refers to how people learn "what to value and to pursue, thus influencing their daily values and decision making" (Segar et al., 2011, p.3). People's stated exercise goals reflect cultural values and social pressures (Segar et al., 2011, p.2). Popular media, public health messaging, and medical professionals are all significant sources of socialization (Segar et al., 2011).

\section{Fatness as a Measure of Health}

Body Mass Index. Body Mass Index (BMI) is calculated by dividing an individual's body mass in kilograms by the square of their height in meters $\left(\mathrm{kg} / \mathrm{m}^{2}\right)$. The original intent of the formula was to establish the physical characteristics of the "average" man (Eknoyan, 2008). In 1972 the formula, previously known as the "Quetelet Index" after creator Adolphe Quetelet, was renamed the "body mass index" by influential obesity researcher Ancel Keys (Keys, Fidanza, Karvonen, Kimura, \& Taylor, 2014, p. 663). Later it was adopted by the National Institutes of Health and the World Health Organization as a tool for estimating obesity at the population level (Murphy, 2014).

Current classifications of weight status by BMI are shown in Table 1 (Ogden, Kit, Carroll, \& Flegal, 2014). 
Table 1. BMI Weight Classifications

\begin{tabular}{ll} 
BMI $\left(\mathrm{kg} / \mathrm{m}^{2}\right)$ & Weight Classification \\
$<18.5$ & Underweight \\
$18.5<25$ & Normal/healthy \\
$25<30$ & Overweight \\
$30<35$ & Grade 1 obese \\
$35<40$ & Grade 2 obese \\
$\geq 40$ & Grade 3 obese/morbidly obese \\
\hline
\end{tabular}

A significant limitation of BMI is its failure to account for body composition (relative proportion of muscle mass and fat mass). Thus athletes and extremely muscular people may be misclassified as obese, while thin, unfit people with dangerous amounts of visceral fat may fall into the normal BMI category (Murphy, 2014).

Waist Circumference and Normal-Weight Central Obesity. Normal-weight central obesity, as determined by waist circumference (WCirc) or waist-to-hip ratio (WHR), may predict weight-associated mortality risk more accurately than BMI (Romero-Corral et al., 2010). Normal-weight central obesity is associated with cardiometabolic dysregulation, metabolic syndrome, and increased risk for cardiovascular disease (Romero-Corral et al., 2010); men with normal-weight central obesity may demonstrate twice the mortality risk than men who are overweight/obese but with no central obesity (Sahakyan et al., 2015).

Cut points for increased risk are a WCirc of $>40$ inches for men, and $>35$ inches for women (Center for Disease Control and Prevention, 2015c). People defined as obese according to their BMI, but not according to their WCirc, experience the same all-cause mortality risk as those with both normal BMI and WCirc; those defined as obese by their 
WCirc demonstrate increased all-cause mortality risk regardless of BMI status (Tanamas et al., 2016). One study found that as many as $23.5 \%$ of American adults aged 20 and older carried significant risk of for metabolic diseases, despite being in the normal BMI category, while as many as $51.3 \%$ of overweight adults and $31.7 \%$ of obese adults studied were metabolically healthy (Wildman et al., 2008). Independent correlates of healthy metabolic profiles among these overweight and obese adults were higher PA levels and smaller WCirc (Wildman et al., 2008).

Relying on BMI alone to determine who is at risk for metabolic and cardiovascular diseases, without consideration of WCirc/WHR and PA levels, potentially overlooks risk in those individuals who, while normal weight, carry more than average amounts of fat tissue and/or are sedentary (Gommez-Ambrosi et al., 2011; Tomiyama, Hunger, Nguyen-Cuu \& Wells, 2016). Meanwhile, metabolically and cardiovascularly healthy, physically active overweight/obese people (as determined by BMI alone) may be classified as "at risk" (Wildman et al., 2008).

Health at Every Size and Active Embodiment. Proponents of the Health at Every Size (HAES) movement believe that weight is an inaccurate metric for health status. Researchers and HAES advocates Linda Bacon and Lucy Aphramor propose that body acceptance, intuitive eating, and "active embodiment" (building regular, enjoyable physical activity into day to day life without requiring adherence to rigid prescriptions for PA dosage or type) offer a more effective and ultimately successful approach to health than do traditional weight loss programs, and that health promotion, not weight control, is the appropriate focus for public education and outreach (Bacon \& Aphramor, 2011). 


\section{Frames}

"Frames" are useful tools for exploring differing perspectives on complex issues. Frames are "definitions of situations" by which people "organize their experiences and guide their actions" (Saguy, 2013, p. 4). How we frame a phenomenon impacts how we respond to that phenomenon. Sociologist Abigail Saguy's writing on how “obesity frames" and "fat frames" shape discourse around weight and health serves as a useful exploration of how frames function. Saguy identifies multiple frames for discussing fat bodies, the most influential being the public health crisis frame, the medical frame, the personal responsibility frame, and the health at every size frame (Saguy, 2013). Each frame can be described briefly as follows:

Public Health Crisis Frame: Overweight/obesity is a population-level health problem (an epidemic), requiring a collective response, including government intervention.

Medical Frame: Overweight/obesity is an individual medical condition requiring medical intervention.

Personal Responsibility Frame: Overweight/obesity is caused by poor personal choices, specifically poor eating and exercise habits (as opposed to being caused by the social environment or genetics).

Health at Every Size Frame: Overweight/obesity aren't problems; what is problematic is the focus on weight loss and dieting; weight is a poor metric for health status; people can be/become healthy without achieving "normal” weights. (Frederick, Saguy, \& Gruys, 2016; Saguy, 2013).

Frames are not mutually exclusive, and frequently overlap. Most public health 
discussions of the obesity epidemic invoke the first three frames listed above; many will also cite socioeconomic frames, social justice frames, and environmental frames (Saguy, 2013).

How these frames are utilized has significant impact on how those receiving the information respond to that information. Via in-depth interviews, a qualitative research study explored how obese adults understand and apply obesity-related public health campaigns (Lewis et al., 2010). Rather than motivating behavior change, anti-obesity campaigns left fat people feeling like failures. Interviewees reported that anti-obesity messaging is overly simplistic, much of it implying that all it takes to reach a "healthy" weight is to "eat right and exercise." Campaigns that ignore the complex social and cultural factors influencing personal health while stressing the personal responsibility frame may leave the intended audience feeling "blamed and disconnected from the message" (Lewis et al., 2010, p.7). The majority of respondents in the Lewis et al. study expressed a desire to see public health campaigns move away from a focus on weight, and instead focus on healthy lifestyle and the benefits of physical activity.

\section{Physical Activity, Weight Control, and False Hope Syndrome}

A review of scientific literature on the effectiveness of weight loss interventions found that the average weight loss achieved by those seeking to lose weight was limited $5-10 \%$ of initial bodyweight; over time most people not only regained that $5-10 \%$, they actually regained more weight than they lost (Mann et al., 2007). Over time, repeated attempts at weight loss, followed by failure to meet or sustain weight loss goals, can leave people frustrated, discouraged, and hopeless (Polivy \& Herman, 2000). This cycle, 
beginning with "high hopes and expectations of success" and ending with "perceptions of oneself as a failure" has been named "false-hope syndrome" (Polivy \& Herman, 2000, pp. 129-130). In face of repeated bouts of false-hope syndrome, people may eventually give up on attempts at behavior change, as the results promised by medical and public health professionals and popular media fail to materialize (Polivy \& Herman, 2000).

Given the frequency of weight loss failure, the framing moderate PA as a path to weight loss is problematic, potentially leading to reduced disposition to PA. This is especially likely in those with consciousness of weight-related stereotypes:

Stereotypes that overweight people are 'lazy' and 'unmotivated' support personal and social misconceptions that physical activity is for thin people, thus perpetuating personally imposed barriers to participation among people who are sensitive to the stigmata assigned people based on weight. Lacking the self-confidence or belief that one has the power and ability to participate in activities linked to weight management is likely to negatively affect perceived competence, and subsequently the pursuit and adoption of physical activity.

(Schmalz, 2010, p. 16)

Experiences of weight based stigma have been associated with avoidance of exercise, reduced motivation to exercise in public (Lewis, et al. 2011; Vartanian \& Novak, 2011), and reduced calorie expenditure through PA (Wott \& Carels, 2010). Additionally, people who are aware of negative stereotypes and stigma against overweight and obese people may experience reduced motivation to engage in PA; this 
reduced motivation in the face of stigma consciousness can occur regardless of the subject's objective weight status (Schmalz, 2010). Schmalz's findings suggest potential negative consequences of health campaigns focused on weight-related goals, as these campaigns may reduce motivation towards PA in both those people who are sensitive about their weight, and in those who are simply aware of weight stigma (without necessarily experiencing it themselves).

\section{Summary}

Inherent in promoting weight loss/weight control as the primary benefit of PA are several risks: 1) normal weight people, including those with normal-weight central adiposity, will conclude that as they do not need to lose weight, they don't need to exercise; 2) people with stigma consciousness will disengage from the message, regardless of their individual weight status; 3) overweight and obese people who have experienced false-hope syndrome and/or who feel stigmatized about their weight will disengage from the message; 4) metabolically and cardiovascularly healthy fat people will feel stigmatized by campaigns that single out their body type as problematic, which could lead to eventual disengagement. The framing of weight control as a primary benefit of PA has the potential to reduce disposition towards PA among people of all weight categories, thus all categories risk losing out on the myriad of non-weight related benefits PA confers. 


\section{Chapter III}

\section{Methods}

\section{Study Design}

This was an exploratory, descriptive study consisting of an anonymous online survey that ran March 5-31, 2018. Respondents rated statements exemplifying three PA frames in terms of how the statement impacted their motivation to engage in PA, and how credible (accurate and trustworthy) they believed the statement to be. Respondents provided anthropometric data, PA data, and information on their utilization of fitness-, health-, and/or wellness-oriented websites.

\section{Participants}

The survey was open to all adults over 18 years of age. Respondents were recruited via social media (websites, blogs, Facebook); recruitment literature included a request that respondents forward the recruitment materials in their own social media network (snowball sampling). In an attempt to avoid overt bias towards a specific frame, recruitment focused on social sites that did not a have a specific health, wellness, or weight loss focus. Human Subjects approval for this study was granted by the PSU Human Subjects Research Review Committee (Appendix A).

\section{Physical Activity Frames}

Informed by Saguy's fat frames, three PA frames were conceptualized:

Weight Control Frame (WCF): A primary benefit of PA is to maintain/obtain a healthy weight; follows conventional PA dosage recommendations.

Example: "Exercise can help prevent excess weight gain or help maintain weight loss. 
When you engage in physical activity, you burn calories. The more intense the activity, the more calories you burn" (Mayo Clinic, 2016).

Medical Frame (MF): A primary benefit of PA is improved health outcomes; follows conventional PA dosage recommendations.

Example: "Regular physical activity can reduce your risk of developing type 2 diabetes and metabolic syndrome...Research shows that lower rates of these conditions are seen with 120 to 150 minutes ( 2 hours to 2 hours and 30 minutes) a week of at least moderateintensity aerobic activity" (CDC, 2015a).

Active Embodiment Frame (AEF): Physical activity should be folded into daily life via enjoyable activities that promote well-being and self-care; benefits of active embodiment are independent of weight loss. The AEF does not follow conventional PA dosage recommendations.

Example: "Exercise and physical activity can be enjoyable. It gives you a chance to unwind, enjoy the outdoors or simply engage in activities that make you happy" (Mayo Clinic, 2016).

For the purposes of this study, responses to the WCF were of primary interest; responses to the AEF in comparison to the WCF was of secondary interest. The MF was included in the study to function as a buffer, out of concern that if only the WCF and AEF were included in the study, the dichotomous nature of the frames would expose the study's intent. The MF was selected for this role based on its prominence in PA promotion and messaging. 


\section{General Procedures}

Online PA promotions and campaigns were identified. Sources were national public health and/or academic institutions, as well as popular, mainstream sites where people seek generalized health information (e.g., Centers for Disease Control, Mayo Clinic online). Websites focused on fitness for sports performance, elite body building, or advanced fitness goals were not included, nor were sites that overtly market themselves as weight-loss oriented (e.g., Weight Watchers) or sites targeted to a niche audience (e.g., Runner's World). Websites targeting health professionals exclusively were not included.

Representative text, exemplifying either the WCF, the MF or the AEF was identified from the selected sites. Text was limited to 1-2 sentences, and needed to work as a "stand alone" message (that is, did not rely on supporting text or imagery to accurately convey its intent). In a few cases, minor edits were made to the statement so that it worked as a stand alone message, or to remove potentially biasing language (for example "Encouragingly, there's strong evidence that staying active can help people slow down or stave off "middle-age spread"" was changed to "There's strong evidence that staying active can help slow down or stave off 'middle-age spread"').

Because strict AEF were difficult to locate (most incorporated elements of weight control and medical frames into their statements, resulting in a "mixed-frame" message), it was necessary to reach beyond the above listed types of sites to find the required number or active embodiment statements. Thus for two of the active embodiment statements, the writings of a popular weight-neutral fitness author were utilized.

After selection, statement order for the survey was established via a random 
number generator. A complete list of resources for the survey statements can be found in Appendix B; the complete survey can be found in Appendix C.

Survey respondents evaluated each statement on a 5-point Likert-scale. For motivation ratings, respondents were asked to complete the sentence "In terms of motivation, I find this message" on the following scale: 1 = "Highly demotivating", $2=$ "Slightly demotivating", 3 = "Neither demotivating or motivating", 4 = "Slightly motivating", 5 = "Highly motivating". For the credibility ratings, respondents were asked to complete the sentence "In terms of the credibility of this message" on the following scale: 1 = "I highly doubt its credibility", 2 = "I slightly doubt its credibility", 3 = "Neutral/don't know", 4 = "I find it slightly credible", 5 = "I find it highly credible".

Respondents provided demographic and anthropometric data (age, gender, height, weight, waist circumference, if they were currently trying to lose weight, and current patterns of PA) as well as information regarding how often they frequent online health and wellness websites.

Out of concern that some respondents - in particular, those with sensitivity about their size, or with stigma consciousness related to weight — would abandon the survey if participation required they provide anthropometric data, respondents were allowed to skip questions they did wish to answer. For that reason, sample sizes in some tables vary due to missing data.

Respondents were given the opportunity to submit comments on the survey ("Do you have any comments or thoughts you would like to share relative to this survey?"), and were prompted to check a box granting permission for their comments to be quoted. 


\section{Data Analysis}

Statement ratings were established by calculating mean scores received by the statement among the entire sample or among specific stratified groups. Mean represents the average rating the statement received on a 1-5 Likert scale ("not motivating/credible" to "highly motivating/credible").

For the purpose of establishing overall statement rankings, statements were grouped into 3 categories. Statement motivation categories were: "not motivating" (combination of ratings 1 and 2); "neutral" (rating 3); and "motivating" (ratings 4 and 5). Statement credibility categories were: "not credible" (combination of ratings 1 and 2); "neutral" (rating 3); and "credible" (ratings 4 and 5). Statement rankings were calculated based on the percentage of respondents who rated the statements motivating/credible (ratings 4 and 5). In the case of two frames scoring the same percentage, mean rating was used to determine final ranking.

BMI was calculated using self-reported height and weight data. Physical activity levels were calculated by multiplying reported bouts of PA per week by the average number of minutes per bout $(\mathrm{m} / \mathrm{wk})$. PA level was categorized as follows: sedentary $(1)=$ $<60 \mathrm{~min} / \mathrm{wk}$; slightly active $(2)=\geq 60$ to $<150 \mathrm{~min} / \mathrm{wk}$; moderately active $(3)=\geq 150$ to $<300 \mathrm{~min} / \mathrm{wk}$; highly active (4) $\geq 300 \mathrm{~min} / \mathrm{wk}$. 


\section{Chapter IV}

\section{Results}

\section{Demographic and Anthropometric Characteristics}

Sample demographics are shown in Table 2. The survey yielded 81 complete responses (responses in which respondents provided complete responses to the statement rating questions). Respondents skewed older (over 50 years of age) and active (PA levels of 3 or 4 ). Women made up $63 \%$ of the survey sample. BMI levels were relatively equally distributed among normal weight, overweight, and obese categories.

Table 2. Sample Demographics

\begin{tabular}{|c|c|c|}
\hline & $\mathrm{n}$ & $\%$ \\
\hline \multicolumn{3}{|l|}{ Gender } \\
\hline Female & 51 & 63.00 \\
\hline Male & 23 & 28.40 \\
\hline Missing Data & 7 & 8.60 \\
\hline \multicolumn{3}{|l|}{ Age } \\
\hline $18-20$ & 2 & 2.47 \\
\hline $21-29$ & 10 & 12.35 \\
\hline $30-39$ & 12 & 14.81 \\
\hline $40-49$ & 19 & 23.46 \\
\hline $50-59$ & 18 & 22.12 \\
\hline 60 and older & 20 & 24.69 \\
\hline \multicolumn{3}{|l|}{ BMI } \\
\hline $18.5<25$ & 26 & 32.10 \\
\hline $25<30$ & 27 & 33.33 \\
\hline$\geq 30$ & 24 & 29.63 \\
\hline Missing Data & 4 & 4.94 \\
\hline \multicolumn{3}{|l|}{ PA levels } \\
\hline 1 & 20 & 24.70 \\
\hline 2 & 12 & 14.81 \\
\hline 3 & 32 & 39.50 \\
\hline 4 & 17 & 20.99 \\
\hline
\end{tabular}




\section{Statement Rankings}

The overall five highest ranked statements in terms of motivation are shown in

Table 3. The top three highest ranked statements were from the AEF; MF statements

rounded out the top five. The highest ranking WCF statement ("Exercise can help prevent excess weight gain or help maintain weight loss") was the ninth highest ranked statement overall. Of the three lowest ranked statements, two were from the WCF. The lowest ranked statement ("Engaging in physical activity can help you connect with family and friends in a fun social setting") was from the AEF. The complete list of motivation ranked statements can be found in Appendix D.

Table 3. Motivation: Five highest ranked statements

\#

Moving your body more can help you feel more confident, more energetic,

4 stronger, and healthier.

Statement
Being active on a regular basis benefits everyday living. It can help you fall asleep faster, be more energized during the day, and boost concentration, all of which 10 make work and play much more satisfying.

Physical activity stimulates various brain chemicals that may leave you feeling

6 happier and more relaxed.

Regular exercise helps prevent or manage a wide range of health problems and concerns, including stroke, metabolic syndrome, type 2 diabetes, depression, a 36 number of types of cancer, arthritis and falls.

Research shows that doing aerobic, muscle-strengthening and bone-strengthening physical activity of at least a moderately intense level can slow the loss of bone

8 density that comes with age.

\section{$\% \quad$ Mean Frame \\ $86.42 \% \quad 4.28 \quad \mathrm{AE}$}

$85.19 \% \quad 4.26 \quad \mathrm{AE}$

$80.24 \% \quad 4.22 \quad \mathrm{AE}$

$79.01 \% \quad 4.12 \quad \mathrm{M}$

$74.07 \% \quad 3.96 \quad \mathrm{M}$

Note: Percentage represents the proportion of participants who rated the statement to be motivating, 4-5, ("slightly" to "highly") on a 1-5 scale; mean represents the average rating on the same scale. $\mathrm{WC}=$ Weight Control; $\mathrm{M}=$ Medical; $\mathrm{AE}=\mathrm{Active}$ Embodiment.

In terms of credibility, results were mixed (Table 4). The top two statements came from the MF, the next two from the AEF, and the fifth from the WCF. Overall, the WCF performed better in the credibility rankings than it did in the motivation rankings, occupying four of the top ten positions, and none of the bottom three positions. The AEF 
statement "Engaging in physical activity can help you connect with family and friends in a fun social setting" scored the lowest in terms of credibility. The complete list of credibility ranked statements can be found in Appendix E.

Table 4. Credibility: Five highest ranked statements

\#

Statement

(\%) Mean Frame

Research shows that doing aerobic, muscle-strengthening and bone-strengthening physical activity of at least a moderately intense level can slow the loss of bone

9 density that comes with age.

$97.53 \% \quad 4.62 \quad \mathrm{M}$

Regular exercise helps prevent or manage a wide range of health problems and concerns, including stroke, metabolic syndrome, type 2 diabetes, depression, a 37 number of types of cancer, arthritis and falls.

$96.29 \% \quad 4.62 \quad \mathrm{M}$

Being active on a regular basis benefits everyday living. It can help you fall asleep faster, be more energized during the day, and boost concentration, all of which

11 make work and play much more satisfying

$95.06 \% \quad 4.58 \quad \mathrm{AE}$

Moving your body more can help you feel more confident, more energetic,

5 stronger, and healthier.

$92.59 \% \quad 4.49 \quad \mathrm{AE}$

Exercise is helpful for weight loss and maintaining weight loss. Exercise can increase metabolism, or how many calories you burn in a day. It can also help you maintain and increase lean body mass, which also helps increase number of 45 calories you burn each day.

$92.50 \% \quad 4.53 \quad \mathrm{WC}$

Note: Percentage represents the proportion of participants who rated the statement to be credible, 4-5, ("slightly" to "highly") on a $1-5$ scale; mean represents the average rating on the same scale. $\mathrm{WC}=$ Weight Control; $\mathrm{M}=$ Medical; $\mathrm{AE}=\mathrm{Active}$ Embodiment.

\section{Overall Frame Ratings}

In terms of motivation, the entire sample rated the frames as follows: $\mathrm{AEF}=4.0$, $\mathrm{MF}=3.8, \mathrm{WCF}=3.7$. In terms of credibility, the entire sample ratings were: $\mathrm{MF}=4.3$, $\mathrm{WCF}=4.3, \mathrm{AEF}=4.3$. Collectively, the AEF scored highest in motivation, and tied the other two frames for credibility.

Frame Ratings Stratified by PA Levels, Age, and Gender

Table 5 shows statement ratings stratified by PA levels, age, and gender. Across all stratification levels, the AEF received the highest mean ratings for motivation. The WCF 
received the lowest mean ratings among those age 30 yrs and up, among the sedentary and moderately active groups, and among men and women when stratified by gender.

Table 5. Frame ratings, stratified by PA, age, and gender

Frame ratings stratified by PA levels

\begin{tabular}{llllllll} 
& \multicolumn{4}{c}{ Motivation Ratings } & \multicolumn{3}{c}{ Credibility Ratings } \\
PA level & $n$ & WC & $M$ & $A E$ & $W C$ & $M$ & $A E$ \\
1 & 15 & 3.08 & 3.39 & 3.70 & 4.30 & 4.25 & 4.13 \\
2 & 17 & 3.96 & 3.94 & 4.03 & 4.30 & 4.28 & 4.30 \\
3 & 32 & 3.75 & 3.94 & 4.01 & 4.24 & 4.35 & 4.17 \\
4 & 17 & 3.81 & 3.78 & 4.03 & 4.45 & 4.44 & 4.45
\end{tabular}

Frame ratings stratified by age

\begin{tabular}{llllllll} 
& \multicolumn{4}{c}{ Motivation Ratings } & \multicolumn{3}{c}{ Credibility Ratings } \\
Age & $n$ & WC & $M$ & $A E$ & $W C$ & $M$ & $A E$ \\
$18-29$ & 12 & 3.70 & 3.64 & 3.87 & 4.60 & 4.50 & 4.34 \\
$30-39$ & 12 & 3.81 & 4.05 & 4.26 & 4.51 & 4.48 & 4.46 \\
$40-49$ & 19 & 3.46 & 3.64 & 3.74 & 4.16 & 4.28 & 4.06 \\
$50-59$ & 18 & 3.79 & 3.97 & 4.04 & 4.17 & 4.28 & 4.14 \\
60 & 20 & 3.71 & 3.77 & 3.97 & 4.27 & 4.22 & 4.34
\end{tabular}

Frame ratings stratified by gender

\begin{tabular}{llllllll} 
& & \multicolumn{3}{c}{ Motivation Ratings } & \multicolumn{3}{c}{ Credibility Ratings } \\
Gender & $n$ & $W C$ & $M$ & $A E$ & $W C$ & $M$ & $A E$ \\
Female & 52 & 3.69 & 3.79 & 3.96 & 4.25 & 4.28 & 4.20 \\
Male & 23 & 3.69 & 3.75 & 3.88 & 4.42 & 4.44 & 4.32
\end{tabular}

Note: Sample sizes vary slightly due to missing data. PA levels: Sedentary $(1)=<60 \mathrm{~m} / \mathrm{wk}$; slightly active $(2)=\geq 60$ to $<150 \mathrm{~m} / \mathrm{wk}$; moderately active (3) $=\geq 150$ to $<300 \mathrm{~m} / \mathrm{wk}$; highly active (4) $\geq 300 \mathrm{~m} / \mathrm{wk}$. Mean represents the average rating on a 1-5 scale ("not motivating/credible" to "highly motivating/credible"). WC=Weight Control; M=Medical; $\mathrm{AE}=$ Active Embodiment.

Results for credibility were mixed. Among respondents age18-39 yrs, the WCF received the highest mean rating; for those age 40-59 yrs, the MF was rated most credible; those over 60 yrs of age rated the AEF as most credible. Stratified by PA level, 
respondents at PA levels 2 and 4 rated the WCF and AEF as equally credible, while those at level 1 preferred the WCF, and those at level 3 rated the MF most credible. Stratified by gender, both men and women rated the MF most credible, and the AEF least credible.

\section{Frame Ratings Stratified by BMI, WCirc, and Weight Status}

Table 6 shows frame ratings stratified by BMI, WCirc, and weight status (desire to gain, maintain, or lose weight). The AEF received the highest mean ratings for motivation, across all stratification levels except men with a WCirc $>40$ inches (the MF rated highest in this group). The WCF received the lowest mean motivation scores across all stratified groups.

Credibility ratings were mixed across the stratified groups, although the MF outperformed the other frames. Stratified by BMI, the WCF was rated most credible by the normal weight group while the AEF was rated least credible (although the means were very close across the three frames). The overweight group rated the MF most credible; the AEF and WCF frame tied in their second place rating. The combined obese group rated the MF the most credible, the AEF the least credible. When stratified by obesity grade, respondents in the grade 1 obesity category rated the WCF the most credible, and the AEF the least credible; respondents in the grade 2 and 3 obese categories rated the MF most credible, the AEF second most credible, and the WCF third. Stratified by WCirc, most groups preferred the MF, the two exceptions being women with a WCirc $\leq 35$ inches and men with a WCirc $>40$ inches; these groups rated the AEF most highly. Weight status ratings were especially mixed; of note is the finding that for those trying to lose weight, the MF achieved the highest mean rating, the AEF the lowest. 
Table 6. Frame ratings, stratified by BMI, WCirc, and weight $\underline{\text { status }}$

Frame ratings stratified by BMI

\begin{tabular}{llllllll} 
& \multicolumn{4}{c}{ Motivation Ratings } & \multicolumn{3}{c}{ Credibility Ratings } \\
BMI & $n$ & WC & $M$ & $A E$ & $W C$ & $M$ & $A E$ \\
$18.5<25$ & 26 & 3.76 & 3.79 & 4.07 & 4.31 & 4.30 & 4.29 \\
$25<30$ & 27 & 3.65 & 3.91 & 4.01 & 4.39 & 4.46 & 4.39 \\
$\geq 30$ & 24 & 3.58 & 3.71 & 3.78 & 4.15 & 4.21 & 4.08
\end{tabular}

Frame ratings stratified by obesity category

\begin{tabular}{llllllll} 
& \multicolumn{4}{c}{ Motivation Ratings } & \multicolumn{3}{c}{ Credibility Ratings } \\
BMI & $n$ & WC & $M$ & $A E$ & $W C$ & $M$ & $A E$ \\
$30<35$ & 15 & 3.84 & 3.89 & 3.90 & 4.32 & 4.22 & 4.10 \\
$35<40$ & 2 & 3.07 & 3.50 & 3.71 & 3.43 & 4.06 & 3.93 \\
$\geq 40$ & 7 & 3.16 & 3.38 & 3.55 & 3.98 & 4.23 & 4.06
\end{tabular}

Frame ratings stratified by WCirc

\begin{tabular}{llllllll} 
& \multicolumn{4}{c}{ Motivation Ratings } & \multicolumn{3}{c}{ Credibility Ratings } \\
WCirc & $n$ & WC & $M$ & $A E$ & $W C$ & $M$ & $A E$ \\
F $\leq 35 "$ & 22 & 3.60 & 3.72 & 3.91 & 4.19 & 4.20 & 4.27 \\
F $>35 "$ & 5 & 3.17 & 3.35 & 3.57 & 4.20 & 4.33 & 3.86 \\
F no data & 24 & 3.48 & 3.66 & 3.97 & 4.12 & 4.25 & 4.12 \\
M $\leq 40 "$ & 14 & 3.39 & 3.58 & 3.77 & 4.04 & 4.22 & 4.06 \\
$\begin{array}{l}\text { M }>40 ” \\
\text { M no }\end{array}$ & 4 & 3.82 & 3.97 & 3.89 & 4.36 & 4.53 & 4.57 \\
data & 5 & 3.49 & 3.66 & 3.82 & 4.18 & 4.31 & 4.18
\end{tabular}

Frame ratings stratified by weight change status

\begin{tabular}{llllllll} 
& & \multicolumn{4}{c}{ Motivation Ratings } & \multicolumn{3}{c}{ Credibility Ratings } \\
Status & $n$ & $W C$ & $M$ & $A E$ & $W C$ & $M$ & $A E$ \\
Gain & 2 & 3.57 & 3.75 & 3.86 & 4.29 & 4.13 & 4.36 \\
Maintain & 31 & 3.70 & 3.79 & 4.00 & 4.28 & 4.20 & 4.19 \\
Lose & 48 & 3.67 & 3.82 & 3.94 & 4.32 & 4.42 & 4.28
\end{tabular}

Note: Sample sizes vary slightly due to missing data. Mean represents the average rating on a 1-5 scale ("not motivating/credible" to "highly motivating/credible"). WC=Weight Control; $\mathrm{M}=$ Medical; $\mathrm{AE}=$ Active Embodiment. 


\section{Other Results}

Respondents' top three ranked reasons cited for engaging in PA were "health benefits" (80.3\%), weight management $(60.5 \%)$, and "stress management, relaxation" (55.6\%) (respondents could select more than one reason for PA engagement). The two options most exemplifying the Active Embodiment frame were two the three lowest

scoring: "I just enjoy being active" (35.8\%); "spend time with family, friends" $(23.6 \%)$. The lowest ranked option, "sports participation" (12.4\%), falls outside of the frames conceptualized for this study. Walking and hiking were the most frequently cited PA activities, followed by resistance training/strength training, and use of cardio machines.

Collectively, respondents did not visit online health, wellness or fitness websites very often. Only $9.9 \%$ reported visiting these websites regularly; $32.1 \%$ reported occasional visits; $55.6 \%$ reported "rarely" or "never" visiting these sites. In terms of motivation, $37.3 \%$ of respondents reported feeling slightly to highly motivated to engage in PA after visiting these sites; $28.4 \%$ reported no change to their motivation, and $4.93 \%$ reported reduced motivation.

Twenty-four respondents provided commentary in response to the prompt at the end of the survey. Most of these responses could be categorized as friendly sign-offs (e.g., "I need to start getting more exercise!"), as brief descriptions of their personal PA routine, or as general comments about health (e.g., "Stay fit guys, health makes wealth. Peace"). Some provided some commentary or insight into their responses to the various statements; these comments are included in the following discussion. 


\section{Chapter V}

\section{Discussion}

The purpose of this exploratory, descriptive study was to examine how respondents rated statements exemplifying three PA frames in terms of inspiring motivation to engage in PA, and in terms of perceived credibility. My prediction was that PA advocacy that closely links PA to weight control would have reduced efficacy in motivating PA as compared to advocacy taking a weight neutral stance (the active embodiment frame). I also predicted that statements linking PA to weight control would not be rated as credible, especially among respondents who fell into the overweight/obese BMI categories.

The first prediction was supported: In the collective study population, the AEF frame achieved the highest mean motivation rating, while the WCF achieved the lowest. In all but one stratified group, the AEF received the highest mean motivation rating. In overall motivation rankings (percent of the study population who rated specific statements as "slightly" or "highly" motivating), the AEF captured the top three positions, while the highest ranked WCF statement occupied the ninth position. A respondent comment encapsulated this finding: "I often find focusing on weight loss and fitness to be demotivating. Focusing on doing things I enjoy (gardening, hiking, biking) keeps me the healthiest and makes me want to stay fit".

Despite the AEF achieving the highest overall motivation ratings among the three frames, it did not rank highly when respondents reported their reasons to engage in PA. This result appears to support Segar's theory of PA socialization—respondents may not 
have selected AEF statements as a reason to engage in PA because they haven't been socialized to think of PA benefits in active embodiment terms. In the collective study population, and in many stratified groups, the AEF received the lowest ratings regarding credibility. In the words of Segar, et al. (2011), it appears that the AEF may have a "branding" problem.

The second prediction was partially supported. Contrary to the predicted outcome, respondents in the grade 1 obesity category rated the WCF the most credible, and the AEF the least credible. However respondents in the grade 2 and 3 obese categories rated the AEF as more credible than the WCF (the MF was the highest rated among these two groups, and in the overweight group). This partially supports the prediction that heavier people would not endorse the WCF. The preference for the AEF over the WCF may indicate endorsement of and/or socialization around the HAES model in this population.

There was a notable overlap between top statements for motivation and credibility - four out of five of the top ranked statements for motivation were also in the top five ranked statements for credibility. Two of these four statements came from the AEF, two from the MF. The AEF statement "Moving your body more can help you feel more confident, more energetic, stronger, and healthier" was the highest ranked statement in terms of motivation ( $86.4 \%$ of respondents rated it motivating), and the fourth highest ranked in terms of credibility ( $92.6 \%$ rated it credible). The MF statement "Research shows that doing aerobic, muscle-strengthening and bone-strengthening physical activity of at least a moderately intense level can slow the loss of bone density that comes with age" received the highest credibility rating, with $97.5 \%$ of respondents rating it credible. 
It was also the rated the fifth most motivating, with $74.1 \%$ rating it motivating. As $35.8 \%$ $(\mathrm{n}=29)$ of respondents were women over 50 years of age, this message may have had particular salience within the study population.

While $92.5 \%$ of the study sample found the WCF statement "Exercise is helpful for weight loss and maintaining weight loss. Exercise can increase metabolism, or how many calories you burn in a day. It can also help you maintain and increase lean body mass, which also helps increase number of calories you burn each day" to be credible, only $63 \%$ rated it as motivating. This exemplifies the "credibility/motivation gap" that was seen throughout the results—credibility scores were higher overall than were motivation scores. This gap was most pronounced in the WCF, with four of the top five largest gaps coming from this frame. The WCF statement "Both diet and physical activity play a critical role in controlling your weight. You gain weight when the calories you burn, including those burned during physical activity, are less than the calories you eat or drink" received a $91.4 \%$ credibility score, and was the second highest ranked WCF statement overall in terms of credibility; however only $50 \%$ of respondents rated it motivating. The credibility/motivation gap was least pronounced in AEF, with four of the five smallest gaps occurring with AEF statements. See Appendix F for the complete list of the credibility/motivation gap rankings.

The WCF credibility/motivation gap may originate in a combination of failed weight loss attempts (Mann et al., 2007), false-hope syndrome (Polivy \& Herman, 2000), and PA socialization (Segar et al,. 2011). People who are socialized to to accept the WCF of PA, but who have not experienced personal success in utilizing PA as a weight loss or 
weight control tool may be able to accept the WCF on a population level (thus finding it credible), while not believing it will work for them on an individual level (thus rating it not motivating).

At first glance, the smaller credibility/motivation gaps seen in the AEF may be viewed as a positive. However, this smaller gap may reflect skepticism regarding the credibility of the frame - if the AEF had received higher credibility ratings, the gaps would have been greater. As noted earlier, PA socialization likely played a role in the overall credibility ratings the AEF received.

While it was not a primary focus of this study, a few comments should be made about the performance of the MF. While outperformed by the AEF in terms of motivation, the MF was rated more motivating than the WCF. In terms of credibility, the MF achieved the highest ratings in the collective study sample, and among adults age 4059 yrs, overweight and most obese respondents, and among those trying to lose weight. Additionally, "health benefits" was the highest ranked reported reason for engaging in PA, with $80.25 \%$ of respondents selecting this option.

The MF also generated the most comments, many of them noting that the respondent did not find MF statements motivating (in some cases, the respondents simultaneously stated a preference for the AEF):

"Descriptions of metabolism and factoids don't motivate me. I already know how exercise and calories work. I don't care that it'll lower my triglycerides or prevent bone density loss. I care about being able to walk up stairs, lift groceries, or play with children." 
"It was interesting, that when the questions were geared scientifically, my brain turned off. When they talked about feeling powerful or empowered, my brain kicked in."

"People statistically do not care about health. Just like they disregard warning signs on cigarettes. They know bad habits are leading them to bad health, but they continue anyway. If you want to make people healthier, show them how activity can be fun or pleasurable."

"The 'avoiding death' arguments are the most unmotivating, and I think such language misinforms people about the real issue with aging — quality of life, mobility, and level of suffering. Living longer per se is not necessarily a good thing or the outcome I think people really want."

"I get more exercise than just about anyone I know, and my HDL/triglycerides have gone up since I started doing this...Luckily, my motivation has absolutely nothing to do with health benefits, but if those benefits had been promised, I'd be highly demotivated and disappointed."

These responses may speak to a credibility/motivation gap within this frame, and offer a suggestion for future studies. Taken as whole, however, the MF performed well in terms of both motivation and credibility, and its out-performance of the WCF among overweight and obese respondents, and among respondents trying to lose weight, has implications for its use in PA promotions targeting those populations.

It is notable that women who opted not to provide their waist circumference were the largest subgroup in the WCirc stratification $(n=24)$. Based on BMI, 13 of those women were obese, 6 were overweight, 3 were normal weight, and 2 did not provide 
height/weight data to establish BMI. While it would be easy to assume that stigmaconsciousness or sensitivity around weight factored into this result, it would be inappropriate to do so based on the data available - perhaps a large percentage of women simply did not have a tape measure at hand. In terms of future studies, this finding may indicate that self-reported WCirc is a difficult data point to capture.

\section{Limitations and Future Research}

The sample skewed toward middle-aged and older, with $46.8 \%$ of respondents over 50 years of age. A younger sample may have yielded different results.

The sample was active, with $60.5 \%$ of respondents meeting or exceeding federal recommendations for PA. In this respect, it was not representative of the United States population. Performing a similar study with a study population consisting of only those people who do not meet PA recommendations would be useful.

The sample size for the graded obese categories was very small, therefore findings may not be representative of those populations.

The frame statements were selected by the principle investigator, based on assumptions of what websites people are familiar with and might frequent, and on those cited in the news media when reporting health information. Future studies should utilize market research to select those sites that are most frequented by the population of interest (older adults, sedentary adults, etc.), and selecting statements from those sites to test. Overall, the study sample did not utilize online health/wellness/fitness websites regularly, thus my selected statements may not be the messages they are used to receiving. 
As seen in Segar's work, motivation does not necessarily lead to participation. My study did not examine actual PA patterns. Further studies are needed to explore the relationship between frames identified as motivating and actual engagement in PA.

\section{Conclusion}

The results of this study suggest that while people may rate the WCF of PA credible, it may not be particularly motivating. This indicates that despite its in dominance in public health and popular media messaging, the WCF may not be the optimal frame for promoting and encouraging PA. The study sample, both collectively and stratified by various demographic and anthropometric characteristics, found the AEF frame motivating, but had mixed responses regarding its credibility; additionally, the AEF did not rank highly in respondents' reported motivations to engage in PA. This may be an indication that socialization around the quality of life enhancing benefits of PA is lacking.

Taken as a whole, these findings suggest two modifications to current PA messaging: 1) a move away from the dominance of the WCF; 2) an increased utilization and promotion of the AEF. These modifications have the potential to address the four risks of WCF focused PA messaging cited earlier in this paper (p. 12), while also addressing the current limitations in the socialization around the AEF. This is not to suggest that the WCF should be completely abandoned - for some the study population, it was both motivating and credible, and therefore likely to be effective. Important caveats to utilization of the WCF are that stigmatizing language be avoided, and acknowledgment that weight/BMI alone is not an accurate metric for health. Ultimately, a 
multi-frame approach that includes all three frames in relatively equal measure may have greater success in motivating PA than does the current, WCF dominant approach. 


\section{References}

Bacon, L., \& Aphramor, L. (2011). Weight Science: Evaluating the evidence for a paradigm shift. Nutrition Journal, 10, 69.

Centers for Disease Control and Prevention. (2014). Facts about physical activity. Retrieved from https://www.cdc.gov/physicalactivity/data/facts.htm

Centers for Disease Control and Prevention. (2015a). The Benefits of Physical Activity. Retrieved from https://www.cdc.gov/physicalactivity/basics/pa-health/index.htm

Center for Disease Control and Prevention. (2015b). The health effects of overweight and obesity. Retrieved from: https://www.cdc.gov/healthyweight/effects/index.html

Center for Disease Control and Prevention. (2015c). Healthy weight. Retrieved from https://www.cdc.gov/healthyweight/assessing/index.html

Eknoyan, G. (2008). Adolphe Quetelet (1796-1874) - The average man and indices of obesity. Nephrology Dialysis Transplantation, 23(1), 47-51.

Frederick, D., Saguy, A., \& Gruys, K. (2016). Culture, health, and bigotry: How exposure to cultural accounts of fatness shape attitudes about health risk, health policies, and weight-based prejudice. Social Science \& Medicine, 165, 271-279.

Goffman, E. (1963). Stigma; notes on the management of spoiled identity. (Spectrum book). Englewood Cliffs, N.J.: Prentice-Hall.

Gómez-Ambrosi, J., Silva, C., Galofré, J., Escalada, J., Santos, S., Millán, D., . . . Frühbeck, G. (2011). Body mass index classification misses subjects with increased cardiometabolic risk factors related to elevated adiposity. International Journal of Obesity, 36(2), 286. 
Keys, A., Fidanza, F., Karvonen, M., Kimura, N., \& Taylor, H. (2014). Indices of relative and obesity. International Journal of Epidemiology, 43(3), 655-665.

Lewis, S., Thomas, S., Hyde, J., Castle, D., Blood, R., \& Komesaroff, P. (2010). "I don't eat a hamburger and large chips every day!" A qualitative study of the impact of public health messages about obesity on obese adults. BMC Public Health, 10, 309.

Lewis, S., Thomas, S., Blood, R., Castle, D., Hyde, J., \& Komesaroff, P. (2011). How do obese individuals perceive and respond to the different types of obesity stigma that they encounter in their daily lives? A qualitative study. Social Science \& Medicine, 73(9), 1349-1356.

Mann, T., Tomiyama, A., Westling, E., Lew, A., Samuels, B., \& Chatman, J. (2007). Medicare's search for effective obesity treatments - Diets are not the answer. American Psychologist, 62(3), 220-233.

Mayo Clinic. (2016). Exercise: 7 benefits of regular physical activity. Retrieved from http://www.mayoclinic.org/healthy-lifestyle/fitness/in-depth/exercise/art20048389/

Murphy, S. (2014). Survival of the fattest $\mid$ why most of we know about obesity may be wrong. New Scientist, 222(2967), 44-47.

Ogden, C., Kit, B., Carroll, M., \& Flegal, K. (2014). Prevalence of childhood and adult obesity in the United States, 2011-2012. JAMA, The Journal of the American Medical Association, 311(8), 806.

Office of Disease Prevention and Health Promotion. (2018). Healthy People 2020: 
Physical Activity Objectives. Retrieved from https://www.healthypeople. gov/2020/topics-objectives/topic/physical-activity/objectives

Polivy, J., \& Herman, C. (2000). The False-Hope Syndrome. Current Directions in Psychological Science, 9(4), 128-131.

Powers, S.K., \& Howley, E.T. (2012). Exercise physiology: Theory and application to fitness and performance ( $8^{\text {th }}$ ed.). New York: McGraw-Hill.

Puhl, R., Moss-Racusin, C., Schwartz, M., \& Brownell, K. (2007). Weight stigmatization andbias reduction: Perspectives of overweight and obese adults. Health Education Research, 23(2), 347-358.

Puhl, R., Peterson, J. L., \& Luedicke, J. (2012). Fighting obesity or obese persons? Public perceptions of obesity-related health messages. International Journal of Obesity, 37(6), 774-782.

Rochholz, D., \& Zidon, M. (2003). Age, Sex, and Socioeconomic Status: Related Factors in Motivations for Exercise, ProQuest Dissertations and Theses.

Romero-Corral, A., Somers, V., Sierra-Johnson, J., Korenfeld, Y., Boarin, S., Korinek, J., . . Lopez-Jimenez, F. (2010). Normal weight obesity: A risk factor for cardiometabolic dysregulation and cardiovascular mortality. European Heart Journal, 31(6), 737-746.

Saguy, A. (2013). What's wrong with fat? New York: Oxford University Press.

Sahakyan, Karine R., Somers, Virend K., Rodriguez-Escudero, Juan P., Hodge, David O., Carter, Rickey E., Sochor, Ondrej, . . Lopez-Jimenez, Francisco. (2015). Normal-weight central obesity: Implications for total and cardiovascular 
mortality. Annals of Internal Medicine, 163(11), 827-35.

Schmalz, D. (2010). 'I Feel Fat': Weight-Related Stigma, Body Esteem, and BMI as Predictors of Perceived Competence in Physical Activity. Obesity Facts, 3(1), 1521.

Schuler, P., Broxon-Hutcherson, A., Philipp, S., Ryan, S., Isosaari, R., \& Robinson, D. (2004). Body-shape perceptions in older adults and motivations for exercise. Perceptual and Motor Skills, 98(3_suppl), 1251-1260.1.

Segar, M., Eccles, J., \& Richardson, C. (2011). Rebranding exercise: Closing the gap between values and behavior. The International Journal of Behavioral Nutrition and Physical Activity, 8, 94.

Shanks, N. (2017). Lift like a girl: Be more, not less. [E-book]. Lioncrest Publishing.

Sherwood, N., \& Jeffery, R. (2000). The behavioral determinants of exercise: Implications for physical activity interventions. Annual Review of Nutrition, 20, 21-44.

Tanamas, S., Ng, W., Backholer, K., Hodge, A., Zimmet, P., \& Peeters, A. (2016). Quantifying the proportion of deaths due to body mass index- and waist circumference-defined obesity. Obesity, 24(3), 735-742.

Tomiyama, A., Hunger, J., Nguyen-Cuu, J., \& Wells, C. (2016). Misclassification of cardiometabolic health when using body mass index categories in NHANES 2005-2012. International Journal of Obesity, 40(5), 883-6.

Trujillo, K., Brougham, M., \& Walsh, R. (2004). Age differences in reasons for exercising. Current Psychology, 22(4), 348-367. 
United States Department of Health Human Services. (2008). 2008 physical activity guidelines for Americans: Be active, healthy, and happy!. (ODPHP publication; no. U0036). Washington, DC]: U.S. Dept. of Health and Human Services.

Vartanian, L., \& Novak, S. (2011). Internalized societal attitudes moderate the impact of weight stigma on avoidance of exercise. Obesity (Silver Spring, Md.), 19(4), 757 62.

Wildman, R., Muntner, P., Reynolds, K., Mcginn, A., Rajpathak, S., Wylie-Rosett, J., \& Sowers, M. (2008). The obese without cardiometabolic risk factor clustering and the normal weight with cardiometabolic risk factor clustering: Prevalence and correlates of 2 phenotypes among the US population (NHANES 1999-2004). Archives of Internal Medicine, 168(15), 1617-24.

World Health Organization. (2018). Obesity and Overweight. Retrieved from http://www.who.int/mediacentre/factsheets/fs311/en/

Wott, C., \& Carels, R. (2010). Overt weight stigma, psychological distress and weight loss treatment outcomes. Journal of Health Psychology, 15(4), 608-14. 


\section{Appendix A-Human Subjects Approval}

\section{P Portland $\underset{\text { UNIVERSITY }}{\text { State }}$}

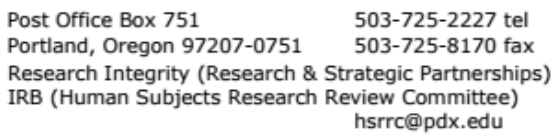

hsrrc@pdx.edu

Date: February 13, 2018

To: Gary Brodowicz / Kristin Spurkland, School of Community Health

From: Lindsey Wilkinson, IRB Chair

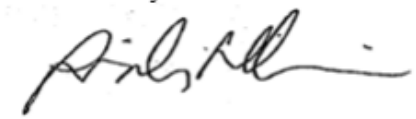

Re: IRB review determination for your protocol \# 184525, entitled: "Framing Physical Activity."

Notice of IRB Review and Determination - Initial Review Exempt Review Category 2; as per Title 45 CFR Part 46

In accordance with your request, the PSU Research Integrity office, on behalf of the IRB (Human Subjects Research Review Committee), has reviewed and approved your protocol for compliance with PSU policies and DHHS regulations covering the protection of human subjects. Research Integrity has determined your protocol qualifies for exempt review and is satisfied that your provisions for protecting the rights and welfare of all subjects participating in the research are adequate. Please note the following requirements:

Approval: You are approved to conduct this research study after receipt of this approval letter, and the research must be conducted according to the plans and protocol submitted (approved copy enclosed).

Consent: You must use IRB-approved consent materials with study participants. Signed consent waived; a written consent statement is required.

Changes to Protocol: Any changes in the proposed study, whether to procedures, survey instruments, consent forms or cover letters, must be outlined and submitted to Research Integrity immediately. The proposed changes cannot be implemented before they have been reviewed and approved by Research Integrity.

Adverse Reactions and/or Unanticipated Problems: If any adverse reactions or unanticipated problems occur as a result of this study, you are required to notify Research Integrity office within 5 days of the event. If the issue is serious, approval may be withdrawn pending an investigation by the IRB.

Completion of Study: Please notify Research Integrity as soon as your research has been completed. Study records, including protocols and signed consent forms for each participant, must be kept by the investigator in a secure location for three years following completion of the study (or per any requirements specified by the project's funding agency).

If you have questions or concerns, please contact the Research Integrity office in Research \& Strategic Partnerships at hsrrc@pdx.edu or call 503-725-2227. 


\section{Appendix B-Statement Sources}

When losing weight, more physical activity increases the number of calories your body uses for energy or 'burns off.' The burning of calories through physical activity, combined with reducing the number of calories you eat, creates a "calorie deficit" that results in weight loss. https://www.cdc.gov/healthyweight/physical_activity/index.html

Moving your body more can help you feel more confident, more energetic, stronger, and healthier. Shanks, N. Lift like a girl: Be more, not less.

Physical activity stimulates various brain chemicals that may leave you feeling happier and more relaxed. https:/www.mayoclinic.org/healthy-lifestyle/fitness/in-depth/exercise/art-20048389/

"Research shows that doing aerobic, muscle-strengthening and bone-strengthening physical activity of at least a moderately intense level can slow the loss of bone density that comes with age."

https://www.cdc.gov/physicalactivity/basics/pa-health/index.htm\#ControlWeight

"Being active on a regular basis benefits everyday living. It can help you fall asleep faster, be more energized during the day, and boost concentration, all of which make work and play much more satisfying."

https://www.health.usnews.com/health-care/articles/2017-09-26/the-benefits-of-simply-moving-more

"Regular physical activity can reduce your risk of developing type 2 diabetes and metabolic syndrome. Metabolic syndrome is a condition in which you have some combination of too much fat around the waist, high blood pressure, low HDL cholesterol, high triglycerides, or high blood sugar."

https:/www.cdc.gov/physicalactivity/basics/pa-health/index.htm\#ControlWeight

"Only a few lifestyle choices have as large an impact on your health as physical activity. People who are physically active for about 7 hours a week have a 40 percent lower risk ofdying early than those who are active for less than 30 minutes a week." https://www.cdc.gov/physicalactivity/basics/pa-health/index.htm

"Exercise can help prevent excess weight gain or help maintain weight loss."

https://www.mayoclinic.org/healthy-lifestyle/fitness/in-depth/exercise/art-20048389/

"Both diet and physical activity play a critical role in controlling your weight. You gain weight when the calories you burn, including those burned during physical activity, are less than the calories you eat or drink."

https://www.cdc.gov/physicalactivity/basics/pa-health/index.htm\#ControlWeight

"Engaging in physical activity can help you connect with family or friends in a fun social setting." https://www.mayoclinic.org/healthy-lifestyle/fitness/in-depth/exercise/art-20048389/

"Physical activity decreases fat around the waist and total body fat, slowing the development of abdominal obesity." https://www.hsph.harvard.edu/obesity-prevention-source/obesity-causes/physical-activity-and-obesity/

"You don't have to do high amounts of activity or vigorous-intensity activity to reduce your risk of premature death. You can put yourself at lower risk of dying early by doing at least 150 minutes a week of moderate-intensity aerobic activity." https://www.cdc.gov/physicalactivity/basics/pa-health/index.htm

"Physically active people have a lower risk of colon cancer than do people who are not active." https://www.cdc.gov/physicalactivity/basics/pa-health/index.htm\#ControlWeight

"Obesity results from energy imbalance: too many calories in, too few calories burned. A number of factors influence how many calories you burn each day, among them, age, body size, and genes. But the most variable factor-and the most easily modified-is the amount of activity you get each day."

https://www.hsph.harvard.edu/obesity-prevention-source/obesity-causes/physical-activity-and-obesity/

"Exercise and physical activity can be enjoyable. It gives you a chance to unwind, enjoy the outdoors or simply engage in activities that make you happy."

https://www.mayoclinic.org/healthy-lifestyle/fitness/in-depth/exercise/art-20048389/

"There's strong evidence that staying active can help slow down or stave off "middle-age spread'." https:/www.hsph.harvard.edu/obesity-prevention-source/obesity-causes/physical-activity-and-obesity/

"When I move my body more, I feel proud of what my body can do."

Shanks, N. Lift like a girl: Be more, not less. 


\section{Appendix B-Statement Sources continued}

"Regular exercise helps prevent or manage a wide range of health problems and concerns, including stroke, metabolic syndrome, type 2 diabetes, depression, a number of types of cancer, arthritis and falls."

https://www.mayoclinic.org/healthy-lifestyle/fitness/in-depth/exercise/art-20048389/

"Winded by grocery shopping or household chores? Regular physical activity can improve your muscle strength and boost your endurance."

https://www.mayoclinic.org/healthy-lifestyle/fitness/in-depth/exercise/art-20048389/

"Being active boosts high-density lipoprotein (HDL), or "good," cholesterol and decreases unhealthy triglycerides."

https://www.mayoclinic.org/healthy-lifestyle/fitness/in-depth/exercise/art-20048389/

"Science shows that physical activity can reduce your risk of dying early from the leading causes of death, like heart disease and some cancers.” https://www.cdc.gov/physicalactivity/basics/pa-health/index.htm\#ControlWeight

"Exercise is helpful for weight loss and maintaining weight loss. Exercise can increase metabolism, or how many calories you burn in a day. It can also help you maintain and increase lean body mass, which also helps increase number of calories you burn each day."

https://www.healthline.com/health/exercise-and-weight-loss\#benefits 
Appendix C-Framing Physical Activity Survey

Directions

Read the following statements, and then rate each one in terms of:

- How the statement impacts your motivation to engage in physical activity

- How credible (accurate and trustworthy) you believe the statement to be

1. (Consent and age agreement)

2. When losing weight, more physical activity increases the number of calories your body uses for energy or "burns off." The burning of calories through physical activity, combined with reducing the number of calories you eat, creates a "calorie deficit" that results in weight loss.

In terms of motivation, I find this message:

Highly demotivating

Slightly demotivating

Neither demotivating or motivating

Slightly motivating

Highly Motivating

3. In terms of the credibility of this message:

I highly doubt its credibility

I slightly doubt its credibility

Neutral/don't know

I find it slightly credible

I find it highly credible

4. Moving your body more can help you feel more confident, more energetic, stronger, and

healthier.

In terms of motivation, I find this message:

Highly demotivating

Slightly demotivating

Neither demotivating or motivating

Slightly motivating

Highly Motivating

5. In terms of the credibility of this message:

I highly doubt its credibility

I slightly doubt its credibility

Neutral/don't know

I find it slightly credible 
I find it highly credible

6. Physical activity stimulates various brain chemicals that may leave you feeling happier and more relaxed.

In terms of motivation, I find this message:

Highly demotivating

Slightly demotivating

Neither demotivating or motivating

Slightly motivating

Highly Motivating

7. In terms of the credibility of this message:

I highly doubt its credibility

I slightly doubt its credibility

Neutral/don't know

I find it slightly credible

I find it highly credible

8. Research shows that doing aerobic, muscle-strengthening and bone-strengthening physical activity of at least a moderately intense level can slow the loss of bone density that comes with age.

In terms of motivation, I find this message:

Highly demotivating

Slightly demotivating

Neither demotivating or motivating

Slightly motivating

Highly Motivating

9. In terms of the credibility of this message:

I highly doubt its credibility

I slightly doubt its credibility

Neutral/don't know

I find it slightly credible

I find it highly credible

10. Being active on a regular basis benefits everyday living. It can help you fall asleep faster, be more energized during the day, and boost concentration, all of which make work and play much more satisfying.

In terms of motivation, I find this message:

Highly demotivating

Slightly demotivating 
Neither demotivating or motivating

Slightly motivating

Highly Motivating

11. In terms of the credibility of this message:

I highly doubt its credibility

I slightly doubt its credibility

Neutral/don't know

I find it slightly credible

I find it highly credible

12. Regular physical activity can reduce your risk of developing type 2 diabetes and metabolic syndrome. Metabolic syndrome is a condition in which you have some combination of too much fat around the waist, high blood pressure, low HDL cholesterol, high triglycerides, or high blood sugar.

In terms of motivation, I find this message:

Highly demotivating

Slightly demotivating

Neither demotivating or motivating

Slightly motivating

Highly Motivating

13. In terms of the credibility of this message:

I highly doubt its credibility

I slightly doubt its credibility

Neutral/don't know

I find it slightly credible

I find it highly credible

14. Only a few lifestyle choices have as large an impact on your health as physical activity. People who are physically active for about 7 hours a week have a 40 percent lower risk of dying early than those who are active for less than 30 minutes a week.

In terms of motivation, I find this message:

Highly demotivating

Slightly demotivating

Neither demotivating or motivating

Slightly motivating

Highly Motivating

15. In terms of the credibility of this message:

I highly doubt its credibility

I slightly doubt its credibility 
Neutral/don't know

I find it slightly credible

I find it highly credible

16. Exercise can help prevent excess weight gain or help maintain weight loss.

In terms of motivation, I find this message:

Highly demotivating

Slightly demotivating

Neither demotivating or motivating

Slightly motivating

Highly Motivating

17. In terms of the credibility of this message:

I highly doubt its credibility

I slightly doubt its credibility

Neutral/don't know

I find it slightly credible

I find it highly credible

18. Both diet and physical activity play a critical role in controlling your weight. You gain weight when the calories you burn, including those burned during physical activity, are less than the calories you eat or drink.

In terms of motivation, I find this message:

Highly demotivating

Slightly demotivating

Neither demotivating or motivating

Slightly motivating

Highly Motivating

19. In terms of the credibility of this message:

I highly doubt its credibility

I slightly doubt its credibility

Neutral/don't know

I find it slightly credible

I find it highly credible

20. Engaging in physical activity can help you connect with family or friends in a fun social setting.

In terms of motivation, I find this message:

Highly demotivating

Slightly demotivating 
Neither demotivating or motivating

Slightly motivating

Highly Motivating

21. In terms of the credibility of this message:

I highly doubt its credibility

I slightly doubt its credibility

Neutral/don't know

I find it slightly credible

I find it highly credible

22. Physical activity decreases fat around the waist and total body fat, slowing the development of abdominal obesity.

In terms of motivation, I find this message:

Highly demotivating

Spurkland: Framing Physical Activity Survey 5

Slightly demotivating

Neither demotivating or motivating

Slightly motivating

Highly Motivating

23. In terms of the credibility of this message:

I highly doubt its credibility

I slightly doubt its credibility

Neutral/don't know

I find it slightly credible

I find it highly credible

24. You don't have to do high amounts of activity or vigorous-intensity activity to reduce your risk of premature death. You can put yourself at lower risk of dying early by doing at least 150 minutes a week of moderate-intensity aerobic activity.

In terms of motivation, I find this message:

Highly demotivating

Slightly demotivating

Neither demotivating or motivating

Slightly motivating

Highly Motivating

25. In terms of the credibility of this message:

I highly doubt its credibility

I slightly doubt its credibility

Neutral/don't know 
I find it slightly credible

I find it highly credible

26. Physically active people have a lower risk of colon cancer than do people who are not active.

In terms of motivation, I find this message:

Highly demotivating

Slightly demotivating

Neither demotivating or motivating

Slightly motivating

Highly Motivating

27. In terms of the credibility of this message:

I highly doubt its credibility

I slightly doubt its credibility

Neutral/don't know

I find it slightly credible

I find it highly credible

28. Obesity results from energy imbalance: too many calories in, too few calories burned. A number of factors influence how many calories you burn each day, among them, age, body size, and genes. But the most variable factor-and the most easily modified-is the amount of activity you get each day.

In terms of motivation, I find this message:

Highly demotivating

Slightly demotivating

Neither demotivating or motivating

Slightly motivating

Highly Motivating

29. In terms of the credibility of this message:

I highly doubt its credibility

I slightly doubt its credibility

Neutral/don't know

I find it slightly credible

I find it highly credible

30. Exercise and physical activity can be enjoyable. It gives you a chance to unwind, enjoy the outdoors or simply engage in activities that make you happy.

In terms of motivation, I find this message:

Highly demotivating 
Slightly demotivating

Neither demotivating or motivating

Slightly motivating

Highly Motivating

31. In terms of the credibility of this message:

I highly doubt its credibility

I slightly doubt its credibility

Neutral/don't know

I find it slightly credible

I find it highly credible

32. There's strong evidence that staying active can help slow down or stave off "middleage

spread".

In terms of motivation, I find this message:

Highly demotivating

Slightly demotivating

Neither demotivating or motivating

Slightly motivating

Highly Motivating

33. In terms of the credibility of this message:

I highly doubt its credibility

I slightly doubt its credibility

Spurkland: Framing Physical Activity Survey 7

Neutral/don't know

I find it slightly credible

I find it highly credible

34. When I move my body more, I feel proud of what my body can do.

In terms of motivation, I find this message:

Highly demotivating

Slightly demotivating

Neither demotivating or motivating

Slightly motivating

Highly Motivating

35. In terms of the credibility of this message:

I highly doubt its credibility

I slightly doubt its credibility

Neutral/don't know 
I find it slightly credible

I find it highly credible

36. Regular exercise helps prevent or manage a wide range of health problems and concerns, including stroke, metabolic syndrome, type 2 diabetes, depression, a number of types of cancer, arthritis and falls.

In terms of motivation, I find this message:

Highly demotivating

Slightly demotivating

Neither demotivating or motivating

Slightly motivating

Highly Motivating

37. In terms of the credibility of this message:

I highly doubt its credibility

I slightly doubt its credibility

Neutral/don't know

I find it slightly credible

I find it highly credible

38. Winded by grocery shopping or household chores? Regular physical activity can improve your muscle strength and boost your endurance.

In terms of motivation, I find this message:

Highly demotivating

Slightly demotivating

Neither demotivating or motivating

Slightly motivating

Highly Motivating

39. In terms of the credibility of this message:

I highly doubt its credibility

I slightly doubt its credibility

Neutral/don't know

I find it slightly credible

I find it highly credible

40. Being active boosts high-density lipoprotein (HDL), or "good," cholesterol and decreases unhealthy triglycerides.

In terms of motivation, I find this message:

Highly demotivating

Slightly demotivating 
Neither demotivating or motivating

Slightly motivating

Highly Motivating

41. In terms of the credibility of this message:

I highly doubt its credibility

I slightly doubt its credibility

Neutral/don't know

I find it slightly credible

I find it highly credible

42. Science shows that physical activity can reduce your risk of dying early from the leading causes of death, like heart disease and some cancers.

In terms of motivation, I find this message:

Highly demotivating

Slightly demotivating

Neither demotivating or motivating

Slightly motivating

Highly Motivating

43. In terms of the credibility of this message:

I highly doubt its credibility

I slightly doubt its credibility

Neutral/don't know

I find it slightly credible

I find it highly credible

44. Exercise is helpful for weight loss and maintaining weight loss. Exercise can increase metabolism, or how many calories you burn in a day. It can also help you maintain and increase lean body mass, which also helps increase number of calories you burn each day.

In terms of motivation, I find this message:

Highly demotivating

Slightly demotivating

Neither demotivating or motivating

Slightly motivating

Highly Motivating

45. In terms of the credibility of this message:

I highly doubt its credibility

I slightly doubt its credibility

Neutral/don't know

I find it slightly credible 
I find it highly credible

46. In the past 7 days, have you engaged in physical activity?

Yes

No

I don't know

47. In a typical week, how many days do you engage in physical activity?

I don't regularly engage in physical activity

Once a week

2 to 4 days a week

5 to 7 days a week

48. In a typical week, how many days do you engage in physical activity?

I don't regularly engage in physical activity

Once a week

2 to 4 days a week

5 to 7 days a week

49. On average, what is the duration of a single session of your physical activity?

20 minutes or less

20-40 minutes

40-60 minutes

60-90 minutes

90 minutes or more

Not applicable

50. What physical activities do you engage in? Check all that apply.

Walking, hiking

Jogging, biking

Cardio machines (treadmill, elliptical, bike, stairmill, rowing machine, ski erg)

Group exercise classes

Dancing

Yoga, pilates

Functional fitness

Resistance training, strength training

Martial arts

Team sports

Not applicable/no answer

Other

51. Do you feel you get too much physical activity, too little physical activity, or about the right amount of physical activity?

Much too much 
Somewhat too much

Slightly too much

About the right amount

Slightly too little

Somewhat too little

Much too little

Not applicable/no answer

51. Why do you engage in physical activities? Check all that apply.

Health benefits

Weight management

Aesthetics (build muscle, shape body)

Sports participation

Functional/therapeutic

Spend time with family, friends

Stress management, relaxation

I just enjoy being active

Not applicable/no answer

Other (please specify)

52. Do you visit fitness, health, and/or wellness oriented websites?

(For example, LIVESTRONG.com, New York Times health section, Centers for Disease

Control, myfitnesspal.com, health.com, mayoclinic.com)

Yes, regularly

Occasionally

Rarely

Never

Not applicable/no answer

Other (please specify)

53. How often do you visit these websites?

Several times a week

Once a week

A few times a month

A few times a year

Very rarely

Never

54. After visiting a fitness, health, and/or wellness oriented website, how motivated do you feel to engage in physical activity?

Highly motivated

Slightly motivated

Slightly unmotivated

Highly unmotivated 
Visiting these websites does not effect my motivation to exercise

Not applicable/no answer

Other (please specify)

55. Are you currently:

Attempting to lose weight

Attempting to gain weight

Neither

Not applicable/no answer

Other (please specify)

56. Gender

57. What is your height in feet and inches? (Remove shoes before measuring.)

Feet

Inches

58. What is your current weight in pounds? (Without clothing)

59. What is your waist circumference in inches?

(Measure at the midpoint between top of your hip bone and the bottom of your ribs.)

60. What is your age?

$18-20$

21-29

30-39

40-49

50-59

60 or older

61. Please describe your race/ethnicity

62. Do you have any comments or thoughts you would like to share relative to this survey?

63. Do you grant permission for your comments in question \#62 to be quoted in Kristin Spurkland's master's thesis?

(Only those who grant permission will be quoted; quotes are anonymous and will not be linked to identifying characteristics.)

$\square$ I give my permission for my comments in question \#62 to be quoted.

$\square$ I do not give my permission for my comments in question \#62 to be quoted. 


\section{Appendix D—Statement Rankings, Motivation}

Regular exercise helps prevent or manage a wide range of health problems and concerns, including stroke, 36 metabolic syndrome, type 2 diabetes, depression, a number of types of cancer, arthritis and falls.

Research shows that doing aerobic, muscle-strengthening and bone-strengthening physical activity of at

8 least a moderately intense level can slow the loss of bone density that comes with age.

34 When I move my body more, I feel proud of what my body can do.

$74.07 \% \quad 3.96 \quad \mathrm{M}$

Science shows that physical activity can reduce your risk of dying early from the leading causes of death, 42 like heart disease and some cancers.

Exercise and physical activity can be enjoyable. It gives you a chance to unwind, enjoy the outdoors or 30 simply engage in activities that make you happy.

16 Exercise can help prevent excess weight gain or help maintain weight loss.

Regular physical activity can reduce your risk of developing type 2 diabetes and metabolic syndrome.

Metabolic syndrome is a condition in which you have some combination of too much fat around the

12 waist, high blood pressure, low HDL cholesterol, high triglycerides, or high blood sugar.

Exercise is helpful for weight loss and maintaining weight loss. Exercise can increase metabolism, or how many calories you burn in a day. It can also help you maintain and increase lean body mass, which also

You don't have to do high amounts of activity or vigorous-intensity activity to reduce your risk of premature death. You can put yourself at lower risk of dying early by doing at least 150 minutes a week of 24 moderate-intensity aerobic activity.

Only a few lifestyle choices have as large an impact on your health as physical activity. People who are physically active for about 7 hours a week have a 40 percent lower risk of dying early than those who are 14 active for less than 30 minutes a week.

Physical activity decreases fat around the waist and total body fat, slowing the development of abdominal 22 obesity.

26 Physically active people have a lower risk of colon cancer than do people who are not active.

$56.79 \% \quad 3.62 \quad$ WC

Winded by grocery shopping or household chores? Regular physical activity can improve your muscle 38 strength and boost your endurance.

When losing weight, more physical activity increases the number of calories your body uses for energy or

"burns off." The burning of calories through physical activity, combined with reducing the number of

2 calories you eat, creates a "calorie deficit" that results in weight loss.

Both diet and physical activity play a critical role in controlling your weight. You gain weight when the calories you burn, including those burned during physical activity, are less than the calories you eat or 18 drink.

Being active boosts high-density lipoprotein (HDL), or "good," cholesterol and decreases unhealthy 40 triglycerides.

32 There's strong evidence that staying active can help slow down or stave off "middle-age spread".

Obesity results from energy imbalance: too many calories in, too few calories burned. A number of factors influence how many calories you burn each day, among them, age, body size, and genes. But the most 28 variable factor-and the most easily modified-is the amount of activity you get each day.

20 Engaging in physical activity can help you connect with family or friends in a fun social setting.

Note: Percentage represents the proportion of participants who rated the statement to be motivating, 4-5, ("slightly" to "highly") on a 1-5 scale; mean represents the average rating on the same scale. $\mathrm{WC}=$ Weight Control; $\mathrm{M}=\mathrm{Medical}$ : $\mathrm{AE}=\mathrm{Active}$ Embodiment. 


\section{Appendix E—Statement Rankings, Credibility}

Research shows that doing aerobic, muscle-strengthening and bone-strengthening physical activity of at 9 least a moderately intense level can slow the loss of bone density that comes with age.

Regular exercise helps prevent or manage a wide range of health problems and concerns, including stroke, 37 metabolic syndrome, type 2 diabetes, depression, a number of types of cancer, arthritis and falls.

Being active on a regular basis benefits everyday living. It can help you fall asleep faster, be more energized during the day, and boost concentration, all of which make work and play much more

11 satisfying

5 Moving your body more can help you feel more confident, more energetic, stronger, and healthier.

Exercise is helpful for weight loss and maintaining weight loss. Exercise can increase metabolism, or how many calories you burn in a day. It can also help you maintain and increase lean body mass, which also

45 helps increase number of calories you burn each day.

Both diet and physical activity play a critical role in controlling your weight. You gain weight when the calories you burn, including those burned during physical activity, are less than the calories you eat or 19 drink.

17 Exercise can help prevent excess weight gain or help maintain weight loss.

Regular physical activity can reduce your risk of developing type 2 diabetes and metabolic syndrome. Metabolic syndrome is a condition in which you have some combination of too much fat around the

13 waist, high blood pressure, low HDL cholesterol, high triglycerides, or high blood sugar.

When losing weight, more physical activity increases the number of calories your body uses for energy or "burns off." The burning of calories through physical activity, combined with reducing the number of

3 calories you eat, creates a "calorie deficit" that results in weight loss.

7 Physical activity stimulates various brain chemicals that may leave you feeling happier and more relaxed.

You don't have to do high amounts of activity or vigorous-intensity activity to reduce your risk of premature death. You can put yourself at lower risk of dying early by doing at least 150 minutes a week of 25 moderate-intensity aerobic activity.

Winded by grocery shopping or household chores? Regular physical activity can improve your muscle 39 strength and boost your endurance.

Science shows that physical activity can reduce your risk of dying early from the leading causes of death, 43 like heart disease and some cancers.

Only a few lifestyle choices have as large an impact on your health as physical activity. People who are physically active for about 7 hours a week have a 40 percent lower risk of dying early than those who are 15 active for less than 30 minutes a week

Exercise and physical activity can be enjoyable. It gives you a chance to unwind, enjoy the outdoors or 31 simply engage in activities that make you happy.

Obesity results from energy imbalance: too many calories in, too few calories burned. A number of factors influence how many calories you burn each day, among them, age, body size, and genes. But the most

29 variable factor-and the most easily modified-is the amount of activity you get each day.

Physical activity decreases fat around the waist and total body fat, slowing the development of abdominal 23 obesity.

Being active boosts high-density lipoprotein (HDL), or "good," cholesterol and decreases unhealthy 41 triglycerides.

33 There's strong evidence that staying active can help slow down or stave off "middle-age spread".

35 When I move my body more, I feel proud of what my body can do.

27 Physically active people have a lower risk of colon cancer than do people who are not active.

21 Engaging in physical activity can help you connect with family or friends in a fun social setting.

$\begin{array}{ccc}\text { (\%) } & \text { Mean } & \text { Frame } \\ 97.53 \% & 4.62 & \mathrm{M} \\ & & \\ 96.29 \% & 4.62 & \mathrm{M} \\ & & \\ 95.06 \% & 4.58 & \mathrm{AE} \\ 92.59 \% & 4.49 & \mathrm{AE}\end{array}$

$92.50 \% \quad 4.53 \quad \mathrm{WC}$

$91.36 \% \quad 4.58 \quad$ WC

$91.36 \% \quad 4.46 \quad$ WC

$91.35 \% \quad 4.64 \quad \mathrm{M}$

$90.12 \% \quad 4.49 \quad$ WC

$90.12 \% \quad 4.48 \quad \mathrm{AE}$

$86.42 \% \quad 4.26-\mathrm{M}$

$82.72 \% \quad 4.35 \quad \mathrm{AE}$

$82.71 \% \quad 4.3 \quad \mathrm{M}$

$82.50 \% \quad 4.25 \quad \mathrm{M}$

$81.48 \% \quad 4.26 \quad \mathrm{AE}$

$79.01 \% \quad 4.21 \quad$ WC

$77.78 \% \quad 3.99 \quad$ WC

$76.55 \% \quad 4.12 \quad \mathrm{M}$

$67.91 \% \quad 3.91 \quad$ WC

$65.43 \% \quad 3.93 \quad \mathrm{AE}$

$60.49 \% \quad 3.85 \quad \mathrm{M}$

$56.79 \% \quad 3.74 \quad \mathrm{AE}$

Note: Percentage represents the proportion of participants who rated the statement to be credible, 4-5, ("slightly" to "highly") on a 1-5 scale; mean represents the average rating on the same scale. $\mathrm{WC}=$ Weight $\mathrm{Control}$; $\mathrm{M}=$ Medical; $\mathrm{AE}=\mathrm{Active}$ Embodiment. 


\section{Appendix F-Credibility/Motivation Gap}

Both diet and physical activity play a critical role in controlling your weight. You gain weight when the calories you burn, including those burned during physical activity, are less than the

18-19 calories you eat or drink.

When losing weight, more physical activity increases the number of calories your body uses for energy or "burns off." The burning of calories through physical activity, combined with reducing

2-3 the number of calories you eat, creates a "calorie deficit" that results in weight loss.

Winded by grocery shopping or household chores? Regular physical activity can improve your 38-39 muscle strength and boost your endurance.

Obesity results from energy imbalance: too many calories in, too few calories burned. A number of factors influence how many calories you burn each day, among them, age, body size, and genes. But the most variable factor-and the most easily modified-is the amount of activity you

28-29 get each day.

Exercise is helpful for weight loss and maintaining weight loss. Exercise can increase metabolism, or how many calories you burn in a day. It can also help you maintain and increase

44-45 lean body mass, which also helps increase number of calories you burn each day.

Being active boosts high-density lipoprotein (HDL), or "good," cholesterol and decreases 40-41 unhealthy triglycerides.

16-17 Exercise can help prevent excess weight gain or help maintain weight loss.

Regular physical activity can reduce your risk of developing type 2 diabetes and metabolic syndrome. Metabolic syndrome is a condition in which you have some combination of too much fat around the waist, high blood pressure, low HDL cholesterol, high triglycerides, or high blood 12-13 sugar.

Research shows that doing aerobic, muscle-strengthening and bone-strengthening physical activity of at least a moderately intense level can slow the loss of bone density that comes with

8-9 age.

You don't have to do high amounts of activity or vigorous-intensity activity to reduce your risk of premature death. You can put yourself at lower risk of dying early by doing at least 150

24-25 minutes a week of moderate-intensity aerobic activity.

Only a few lifestyle choices have as large an impact on your health as physical activity. People who are physically active for about 7 hours a week have a 40 percent lower risk of dying early

14-15 than those who are active for less than 30 minutes a week.

Physical activity decreases fat around the waist and total body fat, slowing the development of 22-23 abdominal obesity.

32-33 There's strong evidence that staying active can help slow down or stave off "middle-age spread".

Regular exercise helps prevent or manage a wide range of health problems and concerns, including stroke, metabolic syndrome, type 2 diabetes, depression, a number of types of cancer, 36-37 arthritis and falls.

Science shows that physical activity can reduce your risk of dying early from the leading cause 42-43 of death, like heart disease and some cancers.

Exercise and physical activity can be enjoyable. It gives you a chance to unwind, enjoy the 30-31 outdoors or simply engage in activities that make you happy.

20-21 Engaging in physical activity can help you connect with family or friends in a fun social setting. Physical activity stimulates various brain chemicals that may leave you feeling happier and more 6-7 relaxed.

Being active on a regular basis benefits everyday living. It can help you fall asleep faster, be more energized during the day, and boost concentration, all of which make work and play much

10-11 more satisfying

26-27 Physically active people have a lower risk of colon cancer than do people who are not active.

Moving your body more can help you feel more confident, more energetic, stronger, and 4-5 healthier.

34-35 When I move my body more, I feel proud of what my body can do.
Fr Cred Mot Gap

WC $\quad 91.36 \% \quad 50.00 \% \quad 41.36 \%$

WC $\quad 90.12 \% \quad 50.62 \% \quad 39.50 \%$

$\mathrm{AE} \quad 82.72 \% \quad 50.62 \% \quad 32.10 \%$

WC $\quad 79.01 \% \quad 48.14 \% \quad 30.87 \%$

WC $\quad 92.50 \% \quad 62.96 \% \quad 29.54 \%$

M $\quad 76.55 \% \quad 49.39 \% \quad 27.16 \%$

WC $\quad 91.36 \% \quad 64.20 \% \quad 27.16 \%$

M $\quad 91.35 \% \quad 64.20 \% \quad 27.15 \%$

M $\quad 97.53 \% \quad 74.07 \% \quad 23.46 \%$

M $\quad 86.42 \% \quad 62.96 \% \quad 23.46 \%$

M $\quad 82.50 \% \quad 59.26 \% \quad 23.24 \%$

WC $\quad 77.78 \% \quad 56.79 \% \quad 20.99 \%$

WC $\quad 67.91 \% \quad 48.14 \% \quad 19.77 \%$

M $\quad 96.29 \% \quad 79.01 \% \quad 17.28 \%$

M $\quad 82.71 \% \quad 65.44 \% \quad 17.27 \%$

$\mathrm{AE} \quad 81.48 \% \quad 65.43 \% \quad 16.05 \%$

$\mathrm{AE} \quad 56.79 \% \quad 44.44 \% \quad 12.35 \%$

AE $\quad 90.12 \% \quad 80.24 \% \quad 9.88 \%$

$\mathrm{AE} \quad 95.06 \% \quad 85.19 \% \quad 9.87 \%$

$\begin{array}{llll}\text { M } & 60.49 \% & 50.62 \% & 9.87 \%\end{array}$

AE $\quad 92.59 \% \quad 86.42 \% \quad 6.17 \%$

$\mathrm{AE} \quad 65.43 \% \quad 66.67 \% \quad-1.24 \%$ 